\title{
Effect of Curing Current on Stiffness and Damping Properties of Magnetorheological Elastomers
}

\author{
Norhiwani Hapipi ${ }^{1}$, Saiful Amri Mazlan ${ }^{1}$, Siti Aishah Abdul Aziz ${ }^{1}$, Ubaidillah ${ }^{2,3 *}$, Norzilawati Mohamad ${ }^{1}$, \\ Izyan Iryani Mohamad Yazid ${ }^{1}$, Seung-Bok Choi ${ }^{4}$ \\ ${ }^{1}$ Engineering Materials Structure and Technology Research Laboratory, Malaysia-Japan International Institute of \\ Technology (MJIIT), Universiti Teknologi Malaysia, Malaysia \\ ${ }^{2}$ Mechanical Engineering Department, Faculty of Engineering, Universitas Sebelas Maret, Indonesia \\ ${ }^{3}$ National Center for Sustainable Transportation Technology, Indonesia \\ ${ }^{4}$ Mechanical Engineering Department, Inha University, Korea \\ *Email: ubaidillah_ft@staff.uns.ac.id
}

\begin{abstract}
In this study, the viscoelastic effects of the magnetic field strength imposed for curing process on the stiffness and damping properties of magnetorheological elastomers (MREs) are experimentally investigated. In order to observe the effect, three different samples of MRE are fabricated by imposing curing current of $0.1 \mathrm{~A}, 0.3 \mathrm{~A}$ and $0.5 \mathrm{~A}$ which is equivalent to the magnetic field of $70 \mathrm{mT}, 309 \mathrm{mT}$, and $345 \mathrm{mT}$, respectively. All samples consist of $30 \%$ silicone rubber and $70 \%$ carbonyl iron particles (CIPs) by weight percentages. After observing the morphological images via SEM, the dynamic performances of these samples, such as storage modulus and loss factor are evaluated and compared as a function of the magnetic field intensity or oscillation frequency. It is shown that the sample cured at $0.5 \mathrm{~A}$ exhibits the highest storage modulus in the frequency domain. In addition, MR effects of three samples are identified, and it is found that the sample cured at $0.5 \mathrm{~A}$ shows the highest absolute and relative MR effect.
\end{abstract}

\section{Keywords}

Magnetorheological Elastomers (MRE); Curing current; Storage modulus; Loss factor; MR effect

\section{Introduction}

Magnetorheological (MR) materials such as MR Fluids (MRF), MR gels, and MR elastomers belong to a class of intelligent materials whose properties can be controlled continuously and reversibly by external stimuli of magnetic fields or currents [1]. Due to these unique properties, MR materials offer huge advantages to be used in various engineering fields, especially in automotive components, vibrations controls, and civil engineering. In recent years, there has been increasing interest in MR Elastomer (MRE) over MRF due to some advantages. Firstly, MRE can overcome the sedimentation problem found in MRF as the matrix is replaced by the solid $[2,3]$. Secondly, MRE is wellknown as an analogy in the field-dependent property to MRF; MRE generally operates in the pre-yield region while MRF operates in the post-yield region [4]. The third benefit is that the control range of stiffness (storage modulus) and damping property (loss factor) is higher than MRE. These salient properties have made MRE as a promising smart material applicable to various engineering fields such as vibration absorbers $[5,6]$, actuators $[7,8]$, dampers $[9,10]$, and sensors $[11,12]$. Basically, MRE consists of high saturation carbonyl iron particle embedded in a solid rubber-like matrix [13]. There are two types of MRE; isotropic and anisotropic MRE. Isotropic MRE is featured by the uniform distribution of the particles inside the matrix in all directions, while the anisotropic MRE has strictly aligned particles direction which can be determined for curing process by applying the external magnetic field $[14,15]$. Therefore, the anisotropic MRE can enhance the storage modulus, loss modulus and MR effect in a certain direction [16-19]. Recently, several MREs which exhibit excellent MR effect and hence practical application areas are widening to the field of vibration absorbers, noise reductions, suspension systems, bushing mount and engine mounts $[20,21]$.

In general, the performance of MRE devices depends on the matrix materials and the types of magnetic particles. Mostly used matrix materials are natural rubber, silicone rubber, and polyurethane rubber. Martinez et al. [22] asserted that one of the most common matrix materials is silicone rubber since it allows the particles to disperse homogeneously in the matrix due to the low viscosity. Wang et al. [23] investigated that the resistance of particles to align is reduced when the matrix of MRE is soft, resulting in a higher tendency of the particles to form perfect chains. 
Several studies have been done in order to improve the performance of MRE including the study on the particle's orientation [19, 24], curing conditions [25], also the size and the concentration of particles. For example, Ju et al. [19] fabricated anisotropic polyurethane-based MREs with a constant magnetic field strength of $700 \mathrm{mT}$. The result showed that the MR effect of the anisotropic MRE was $33.7 \%$. Wu et al. [24] discovered that curing the polyurethane MRE under magnetic flux density of $900 \mathrm{mT}$ provided the relative MR effect of $21 \%$. The effect of several parameters, such as the magnetic field strength, time and temperature during the curing process of MRE, was studied by Li et al. [25].

In this work, the variety of magnetic field strengths ranging from 0 to $350 \mathrm{mT}$ were used, showing $64.7 \%$ of MR effect can be achieved by using the optimum magnetic field of $110 \mathrm{mT}$ during the curing process. It concluded that the strength of the magnetic field significantly affects the formation of the chain-like structures, and consequently results in different MRE performances. Thus, the magnetic field strength to be imposed for curing process plays an important role in determining the formation of the chain-like structure, as it consequently affects the overall properties of MRE. In addition to MR effect, it is well known that damping property of MRE is also one of the main parameters to be considered as it has potential applications, such as vibration-damping bearing, damping, and gasket [26].

The effectiveness of MRE to act as vibration absorbers is greatly depends on the damping property of MRE that can be represented by the loss factor [20, 28]. In other words, the damping property is a special characteristic that influences the vibratory of dynamic systems in which vibration control or suppression needs to be done by dissipating the energy in heat form. It is generally known that the optimal level of damping property is required to maximize vibration control efficiency $[27,28]$. Therefore, a study on the variation of the damping property of MRE should be investigated with respect to several parameters.

In this work, among several parameters affecting both the stiffness and damping property, curing current (or magnetic field strength) is chosen to investigate the field-dependent viscoelastic characteristics experimentally. Especially, the change of the damping property due to the different curing current is focused in this work since the research work on this issue is considerably rare. In order to undertake this work, three different MRE samples are fabricated with the different curing current of $0.1 \mathrm{~A}, 0.3 \mathrm{~A}$, and $0.5 \mathrm{~A}$, which is equivalent to the magnetic field of $70 \mathrm{mT}$,
$309 \mathrm{mT}$, and $345 \mathrm{mT}$, respectively. By imposing different magnetic field for the curing process, both MR effect and damping property can be varied due to the interaction between the particles and the matrix. Moreover, to identify the effect due to the curing current only, three samples are fabricated with same matric and particles; $30 \%$ silicone rubber and $70 \%$ carbonyl iron particles (CIPs) by weight percentages.

After observing the chain-like structures through the microphotograph taken from SEM, dynamic properties of MRE samples are tested using the oscillation rheometer. Then, the measured results of storage modulus, loss factor and MR effect are presented as a function of the driving frequency or/and the intensity of the magnetic field. It is identified from the results that the behavior of the storage modulus is simple, showing the higher value with the higher curing current. However, the behavior of the loss factor is very complex exhibiting the lower value with the higher curing current at lower strain, but an opposite behavior at high strain over a certain threshold strain. In addition, it is evaluated that the MR effect is increased as the curing current increases without any complex behavior.

\section{Experimental Setup}

\subsection{Preparation of MRE samples}

The fabrication process of MRE samples consists of two steps; mixing and curing process. The roomtemperature vulcanized RTV-two NS625 (Nippon Steel) silicone rubber, SR was used as the matrix. The German-made BASF spherical CIP with an average diameter of $6 \mu \mathrm{m}$ was used as a magnetic particle. The density for both SR and CIP is $1.08 \mathrm{~g} / \mathrm{cm}^{3}$ and 7.874 $\mathrm{g} / \mathrm{cm}^{3}$ respectively.

The anisotropic MRE was prepared in the presence of different magnetic fields while isotropic MRE was prepared without an external magnetic field to observe the difference between the particle orientations. The CIP with SR were mixed thoroughly using a mechanical stirrer for approximately 20 minutes. The mixture was placed in a vacuum oven to remove the air bubbles. The peroxide liquid was added as curing agents, and the mixture was stirred for another 2 minutes until the solution was homogeneously distributed. After that, the mixture was poured into a customized mold with $1 \mathrm{~mm}$ thickness and oriented under a magnetic field. Three types of MREs samples with 70 wt. $\%$ corresponds to $25 \mathrm{vol} \%$ of CIPs at a different curing current of $0.1,0.3$, and $0.5 \mathrm{~A}$ was prepared as shown in Table 1. 
Table 1 The composition of MRE samples

\begin{tabular}{cccc}
\hline Sample & $\begin{array}{c}\text { Silicone rubber } \\
{[\boldsymbol{w} \boldsymbol{t} . \%]}\end{array}$ & $\begin{array}{c}\text { CIPs } \\
{[\boldsymbol{w} \boldsymbol{t} \% \mathbf{\%}]}\end{array}$ & $\begin{array}{c}\text { Curing current } \\
{[\mathbf{A}]}\end{array}$ \\
\hline Iso & 30 & 70 & 0.0 \\
Aniso-1 & 30 & 70 & 0.1 \\
Aniso-2 & 30 & 70 & 0.3 \\
Aniso-3 & 30 & 70 & 0.5 \\
\hline
\end{tabular}

\subsection{Electromagnetic device for the curing process}

The electromagnetic device was developed to be used during the curing process to fabricate the anisotropic MRE. The schematic diagram of the equipment used for aligning the particles is illustrated in Figure 1. The magnetic distribution of the magnetic device was first determined through the magnetic circuit analysis. The magnetic flux distribution within the magnetic device was then obtained from the Finite Element Method Magnetics (FEMM) software and shown in Figure 2. The result from the FEMM simulation was verified by comparing the simulation results with the actual measurements at the test chamber of the curing device. The strength of the magnetic field can be adjusted by controlling the current of the power supply. The simulation result in Figure 2 proves that the magnetic flux densities throughout the MRE samples are uniformly distributed. Table 2 provides the validation result obtained from the simulation compared to the actual measurement near the electromagnetic device.

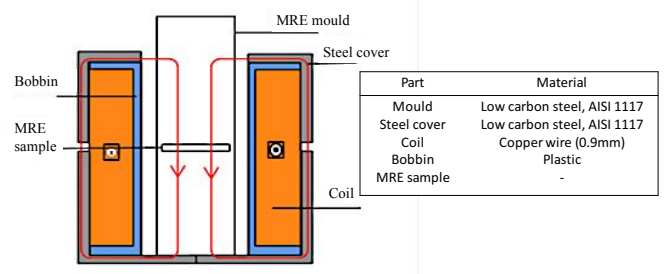

Figure 1 Magnetization equipment used for particles alignment

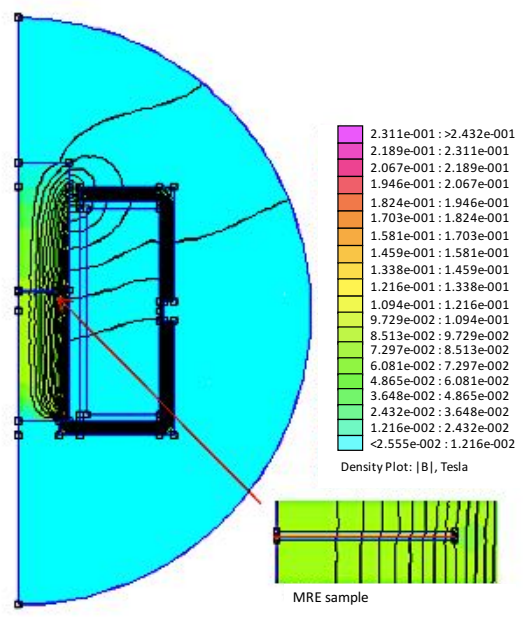

Figure 2 Magnetic field distribution of MRE inside the mold
Table 2 The simulation results of magnetic flux density in the MRE region

\begin{tabular}{cc}
\hline Curing current [A] & Magnetic flux density [mT] \\
\hline 0.0 & 0.0 \\
0.1 & 70 \\
0.3 & 209 \\
0.5 & 345 \\
\hline
\end{tabular}

\subsection{Dynamic oscillatory test setup}

The rheological properties which involve the variation of strain amplitude, excitation frequency, and magnetic field intensity testing condition were performed to measure the dynamic performance of MRE. The rheological properties were measured by using an oscillation Rheometer (Physica MCR 302, Anton Paar, Germany) utilizing a magnetorheological device (MRD170) as the electromagnetic unit. The MRE samples have the sizes of $1 \mathrm{~mm}$ and $20 \mathrm{~mm}$ in thickness and diameter, respectively. Fundamentally, the shear storage modulus $\left(G^{\prime}\right)$ and loss factor $(\tan \delta)$ are the parameters to describe the rheological properties of viscoelastic materials such as MRE. The shear storage modulus can be described as the ability of the material to store the energy during shearing process, while the loss factor is the ratio between storage modulus and loss modulus and serve as the damping properties of the materials. In this work, the rheological properties of MRE as a function of the magnetic field, amplitude strain and oscillation frequency were investigated. In the strain sweep test, the strain ranges from $0.001 \%$ to $10 \%$ with a constant frequency of $1 \mathrm{~Hz}$ while the swept frequency ranges from $0.1 \mathrm{~Hz}$ to $100 \mathrm{~Hz}$ at a constant strain of $0.1 \%$. The storage modulus, $G^{\prime}$, and the loss factor, $\tan \delta$ were measured for all MRE samples in order to obtain the effect of magnetic field on the MRE, the amplitude strain and frequency were experimentally investigated at a constant rate of shear strain of $0.1 \%$ and frequency, $1 \mathrm{~Hz}$. The external magnetic field intensity used varies linearly from $0 \mathrm{mT}$ to $800 \mathrm{mT}$. All the rheological testing was done at room temperature, 25 ${ }^{\circ} \mathrm{C}$.

\section{Results and Discussion}

\subsection{Morphological images}

Figure 3 illustrates the SEM micrographs of SR-based MRE for both isotropic and anisotropic MRE. It is found that from Figure 3 (a), in the isotropic MRE samples, the CIPs are randomly dispersed in the SR matrix as the sample was cured without the magnetic field. However, in the anisotropic MRE samples (Figure 3 (b)), the CIPs form chain-like structures aligned in the direction of the magnetic field (arrow) which is applied during the curing process. The 
formation of chain-like structures results from the interaction force between the magnetized particles. As the strength of the magnetic field is increased, the formation of chain-like structures is expected to be denser as reported by several researchers thus improved the mechanical performance of MREs [29].

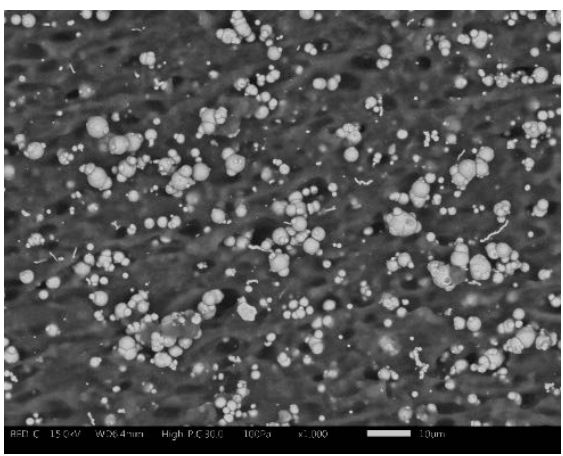

(a)

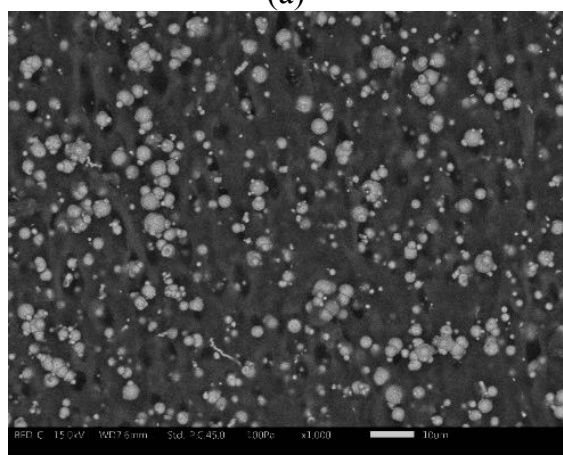

(b)

Figure 3 SEM micrograph images of (a) isotropic and (b) anisotropic (347 mT) MRE with $70 \mathrm{wt} . \%$ of carbonyl iron particles

\subsection{Strain amplitude}

The strain amplitude sweep test was carried out to obtain the dynamic behavior of MRE samples in response to the external magnetic field. Parameters to describe the performance of MRE are known as storage modulus and loss factor which represents the stiffness and damping factor of MREs [30]. Figure 4 depicts the storage modulus as a function of strain amplitude test in the off-state (open symbol) and onstate (closed symbol) conditions. As shown in Figure 4 , the storage modulus of all the samples, isotropic and anisotropic samples decreased with the increasing strain. This phenomenon indicates a strong behavior of the Payne effect [23]. The Payne effect happens due to the destruction and reformation of the particle filler network when subjected to the magnetic field. Particularly, the storage modulus decreased slightly at small strain before approximately $1 \%$ and started to decline drastically after approximately $1 \%$ (critical strain). At a low strain region $(0.001 \%$ to $1 \%)$, the storage modulus is independent on the strain which means that the structures are still in linear viscoelastic (LVE) region and not affected by the external magnetic field [31]. On the other hand, the initial storage modulus, $G_{0}$ of anisotropic samples is higher than the isotropic sample. The value of $G_{0}$ is more pronounced in on-state conditions. As shown, the $G_{0}$ value increased as the strength of the magnetic field strengthened. It is mainly caused by the application of the external magnetic field, which causes all the chainlike particles to induce the magnetic dipole interactions and hinder any deformation during shear loading. As a result, the dynamic stiffness of the MRE increases [32]. By curing the MRE with the stronger magnetic field, the chain-like structures enhance and strengthen the particle-particle interactions as the structures become denser and thicker [33]. Moreover, the stiffness of anisotropic MRE depends on the distances and the particle-particle interaction inside the matrix [30]. As the structures become denser and thicker, the distance between the particles is decreased. Figure 5 presents the strain dependence of the loss factor as a function of strain. Loss factor can be used to determine the damping property of MRE.

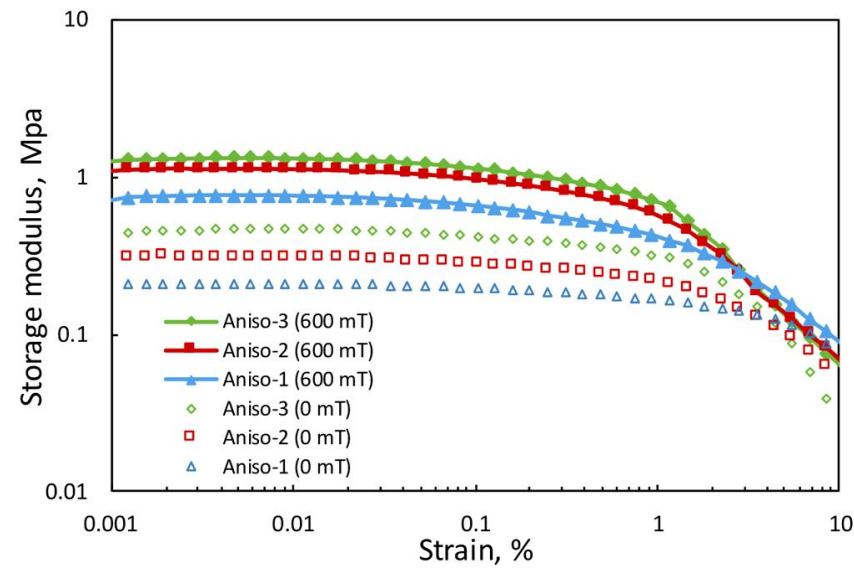

Figure 4 Storage modulus versus strains under magnetic fields of $0 \mathrm{mT}$ and $600 \mathrm{mT}$

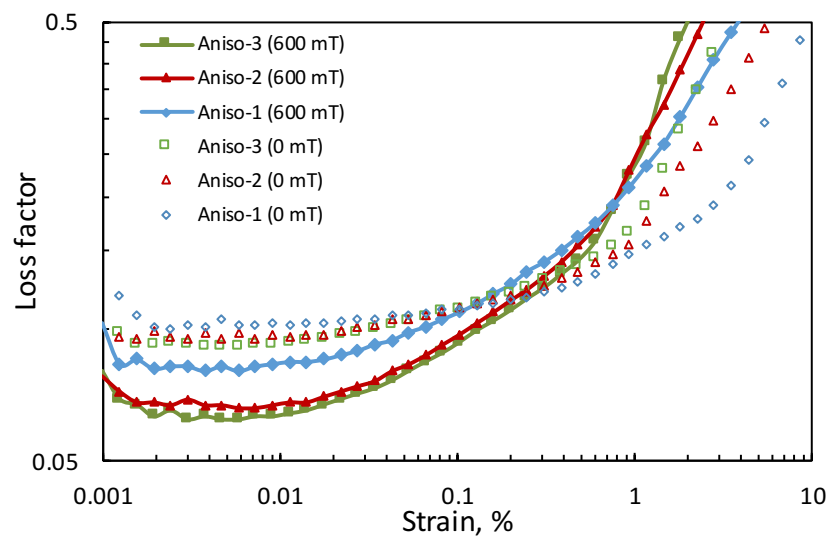

Figure 5 Loss factor versus strains under magnetic fields of $0 \mathrm{mT}$ and $600 \mathrm{mT}$

As illustrated in Figure 5, the trend shows that the loss factor increases parallel with the shear strain amplitude. However, the damping ratio decreases 
when the magnetic strength is increased during the curing process. When higher magnetic field strength is used, the magnetic coupling gets stronger, which reduces the distances between particles and make them closely packed together resulting in less heat dissipated. In addition, Sample 3 has a lower loss factor compared to sample 1 and 2. Stronger network particles require high energy to destruct the network structure. This fact indicates that the higher magnetic flux density applied during the curing process will cause the strong magnetic interactions between particles, to resist the breakage of the network at small strain, thus reducing the energy dissipation under an oscillatory force. In addition, a higher value of loss factor is achieved when the greater degree of molecular mobility is used. All the samples exhibit low damping characteristics when a low percentage of strain is used. The loss factor is at its lowest value when the sample cured by using the strong current of 0.5 A respective to $345 \mathrm{mT}$. The decrease of the loss factor may be caused by a better interaction between the matrix and particles, causing less sliding and slip friction [33]. With the increment of the magnetic flux density, the interaction between two particles becomes stronger, thus resulting in the decrement of the movement of molecular chains and energy dissipation.

\subsection{Oscillation frequency}

The effect of frequency on the storage modulus was tested by using oscillatory shearing at a constant strain of $0.02 \%$. The frequency dependent modulus for all the samples with different curing magnetic strength measured in off-state and on-state condition $(600 \mathrm{mT})$ is shown in Figure 6.

Figure 6 illustrates the storage modulus for all samples, which indicates the stiffness of the material, increases linearly with frequency and magnetic field strength. For sample 1, the storage modulus increases from 0.58 to $0.99 \mathrm{MPa}$ while in sample 2 and 3, the storage modulus increased from 0.93 to $1.43 \mathrm{MPa}$ and from 0.98 to $1.48 \mathrm{MPa}$, respectively. Additionally, the $G_{0}$ of the MREs increased gradually with the increase of magnetic field strength during the curing process. It is because the magnetic forces between particles are strongly influenced by the strength of the magnetic field. The higher the magnetic field, the better the interactions that occur between the particles. Therefore, when the stronger magnetic field is used, the distance of the particles reduced, and the zero-field modulus is enhanced. Furthermore, the $G_{0}$ for all samples, isotropic and anisotropic is higher when tested at on-state (closed symbol) than off-state (open symbol) due to the existence of chain-like structures that increased the stiffness of the MRE samples.
Figure 7 shows the variety of loss factor as a function of the testing frequency. The loss factor can be explained by the increment of energy loss due to the destruction and deformation of the carbonyl iron particles in the silicon matrix.

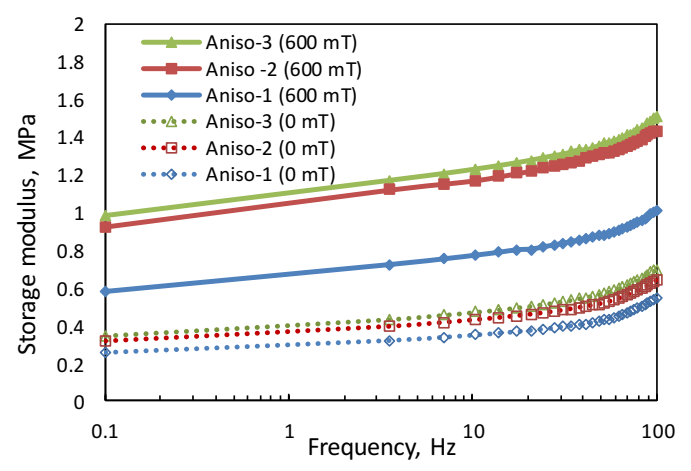

Figure 6 Storage modulus as a function of frequency under magnetic fields of $0 \mathrm{mT}$ and $600 \mathrm{mT}$

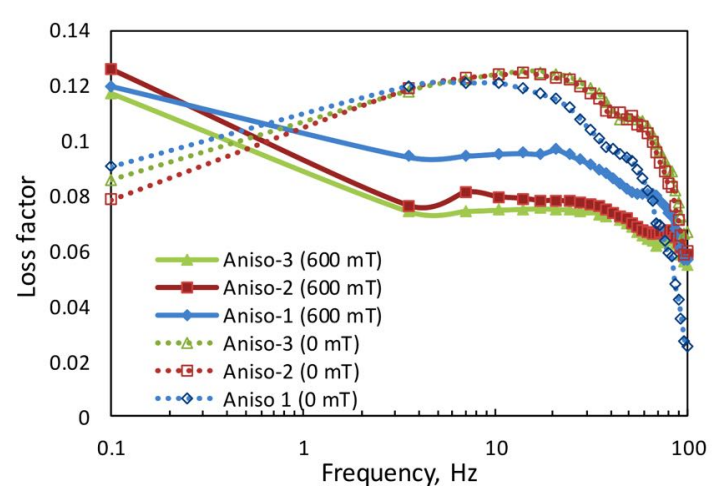

Figure 7 Loss factor as a function of frequency under magnetic fields of $0 \mathrm{mT}$ and $600 \mathrm{mT}$

In Figure 7 the result indicates that, at off-state conditions, the loss factor firstly increased with the increment of frequency before approximately $20 \mathrm{~Hz}$ and then start to decrease gradually with a further increment of frequency approximately after $20 \mathrm{~Hz}$. Whilst, at on-state conditions, the loss factor sharply decreased with the increment of frequency and then started to decrease with a further increment of frequency slightly. This phenomenon is caused by the reaction of the MRE samples toward the external magnetic field. Furthermore, it is noticed that the loss factor if MRE samples decreased as the strength of the magnetic field during curing is increased. This can be explained by the formation of the chain-like structures during the curing process. As the magnetic field strength is stronger, the chain-like structure becomes thicker and stronger which means that the interaction between the particles become stronger thus reducing the friction between the particles [33, 34]. Therefore, as the friction energy between the particles decreases, the energy dissipations is lowered, which results in the decrement of loss factor. 


\subsection{Magnetic field swept}

The key parameter to determine the MRE performance is obtained by measuring the absolute and the relative MR effect of the sample. The absolute MR effect is described by (1), and the relative MR effect is described by (2).

$\Delta G=G_{\text {max }}^{\prime}-G_{0}$

$M R_{\text {effect }}=\left(\Delta G / G_{0}\right) \times 100 \%$

Where, $G^{\prime}{ }_{\max }$ is the storage modulus at magnetic saturation, and $G_{0}$ is the zero-field modulus. The fielddependent shear modulus and the relative MR effect of all the samples are shown in Figure 8 During the testing, the frequency was fixed constant at $1 \mathrm{~Hz}$, and the strain amplitude was $0.02 \%$. Figure 8 shows the dependence of the shear storage modulus of MRE samples on the magnetic field sweep. As can be seen, all the samples exhibit a similar trend where the shear storage modulus increases with the increasing of testing magnetic field up to $800 \mathrm{mT}$. This behavior is very common as once the magnetic field was applied to the MREs, the magnetic dipole moment of the CIPs inside the matrix is induced and formed chain-like structures that restricted the formation of silicone rubber matrix which results in a change of shear modulus. The absolute and relative MR effect of the MREs are calculated and summarized in Table 3.

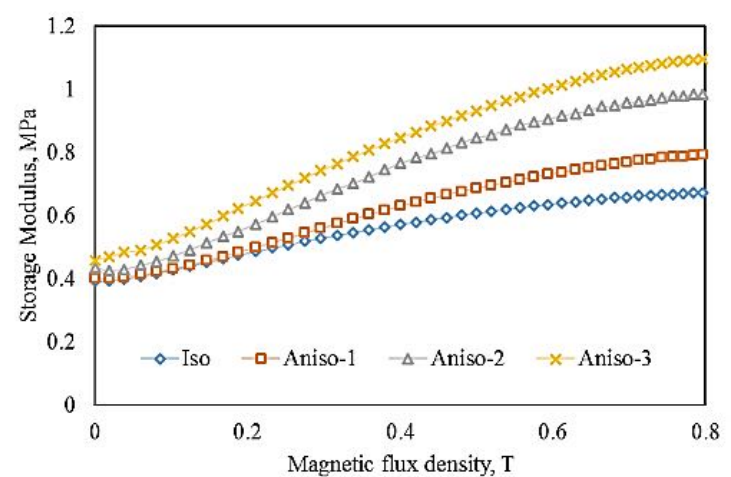

(a)

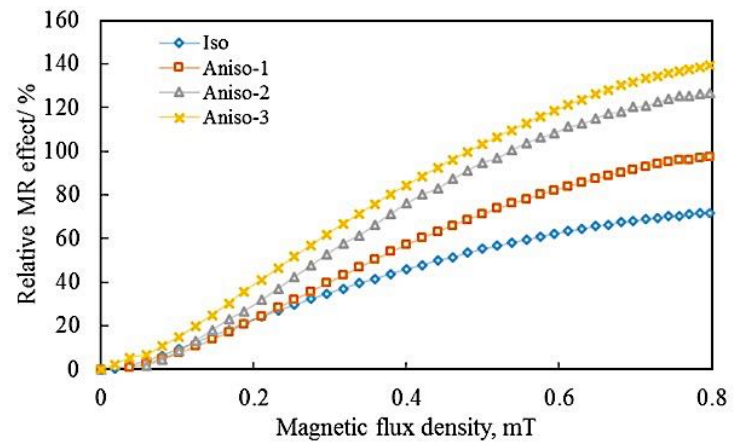

(b)

Figure 8 Magnetic field swept results; (a) shear storage modulus, and (b) relative MR effect the samples
Table 3 The simulation results of magnetic flux density in the MRE region

\begin{tabular}{cccc}
\hline Sample & $\mathbf{G}_{\mathbf{0}} \mathbf{( M P a )}$ & $\boldsymbol{\Delta} \mathbf{G} \mathbf{( M P a )}$ & $\boldsymbol{\Delta} \mathbf{G} / \mathbf{G}_{\mathbf{0}} \mathbf{( \% )}$ \\
\hline Iso-0 & 0.39 & 0.28 & 73 \\
Aniso-1 & 0.40 & 0.39 & 97 \\
Aniso-2 & 0.43 & 0.55 & 125 \\
Aniso-3 & 0.45 & 0.64 & 137 \\
\hline
\end{tabular}

As presented in Table 3, the $G_{0}$ of MRE samples increased steadily with the increasing of curing magnetic field. It is apparent that sample 1 exhibits the lowest zero-field modulus of $0.40 \mathrm{MPa}$ as compared to other MRE samples. Additionally, the MR effect of the samples is proportionally increased to the magnetic field during the curing process. The lowest MR effect achieved by an isotropic sample, Iso-0 which is $73 \%$. The relative MR effect for anisotropic samples, Aniso2, Aniso-3, and Aniso-4, is 97, 125, and $137 \%$ respectively. This increment of the MR effect can be explained by the formation of a thicker and stronger chain when the samples are subjected to the high curing field during the sample preparation. When a higher magnetic field strength is applied during curing, the particles inside the elastomer matrix formed more complex structures and consequently enhanced the MR effect [35]. According to $\mathrm{Li}$ et al., the fabrication of anisotropic MRE with the strong magnetic field during the curing process will form more restrained rubber that is trapped between the particles, which increase the modulus and strengthen the chain-like structure in MRE [25]. The magneto-induced modulus $(\Delta G)$ is also higher for the samples cured at higher curing field due to the strong attraction between the magnetic particles, which cause the particles to be attracted more easily to each other and form a strong, complex structure.

\section{Conclusion}

In this study, the effect of the curing current (or magnetic field) on the field-dependent rheological characteristics of MREs were experimentally investigated, especially focusing on the damping property. As a first step, three different MRE samples were fabricated at three different curing current, while keeping the same matrix and particle percentage. In addition, to observe the different effect, two different types of MRE; isotropic and anisotropic MRE were fabricated with $70 \mathrm{wt}$. \% of CIP. Firstly, it has shown that the anisotropic MRE has a higher MR effect than the isotropic MRE at the same conditions. Secondly, from the dynamic test sweep, it has identified that the higher magnetic field during the curing process results in higher initial shear storage of the MREs. The stiffness and MR effect of the matrix also has been increased when the curing current is increased. The maximum increase of MR effect up to $137 \%$ is 
achieved from the MRE sample fabricated with the highest curing current. Unlike both the storage modulus and MR effect, the behavior of the damping property (or loss factor) exhibits a complex behavior with respect to the curing current. It has been shown that the loss factor of MRE samples is decreased as the curing current increases, but there is a threshold value which indicates different damping behavior. In other words, the behavior of the loss factor is decreased with the higher curing current at lower strain, but an opposite behavior at high strain has occurred. The results presented in this work are very useful to fabricate right MRE for the right application. An optimal MRE possessing desired rheological properties with respect to the magnetic field can be fabricated by choosing an appropriate curing current (magnetic field). It is finally remarked that the detailed analysis of the chain-like mechanism depending on the curing current needs to be undertaken through more sophisticated morphological imaging process in the future.

\section{Acknowledgment}

This work is supported and funded by Professional Development Research University (PDRU) grant (Vot No. 04E02). This work is also partially supported by USAID through Sustainable Higher Education Research Alliances (SHERA) Program - Center for Collaborative (CCR) National Center for Sustainable Transportation Technology (NCSTT) with Contract No. IIE00000078-ITB-1.

\section{References}

[1] J.-H. Koo, F. Khan, D.-D. Jang, and H.-J. Jung, "Dynamic characterization and modeling of magneto-rheological elastomers under compressive loadings," Smart Mater. Struct., vol. 19, no. 11, p. 117002 , Nov. 2010.

[2] M. Kukla, J. Goreeki, I. Malujda, K. Talaska, and P. Tarkowski, "The Determination of Mechanical Properties of Magnetorheological Elastomers (MREs)," Procedia Eng., vol. 177, pp. 324-330, 2017.

[3] X. G. Huang, Z. Y. Yan, C. Liu, G. H. Li, and J. Wang, "Study on the resistance properties of magnetorheological elastomer," Mater. Res. Innov., vol. 19, no. sup5, pp. S5924-S5-928, May 2015.

[4] Ubaidillah, J. Sutrisno, A. Purwanto, and S. A. Mazlan, "Recent Progress on Magnetorheological Solids: Materials, Fabrication, Testing, and Applications," Adv. Eng. Mater., vol. 17, no. 5, pp. 563-597, May 2015.

[5] H.-X. Deng and X.-L. Gong, "Application of magnetorheological elastomer to vibration absorber," Commun. Nonlinear Sci. Numer. Simul., vol. 13, no. 9, pp. 1938-1947, Nov. 2008.

[6] H. K. Kim, H. S. Kim, and Y.-K. Kim, "Stiffness control of magnetorheological gels for adaptive tunable vibration absorber," Smart Mater. Struct., vol. 26, no. 1, p. 015016, Jan. 2017.

[7] J.-H. Koo, A. Dawson, and H.-J. Jung, "Characterization of actuation properties of magnetorheological elastomers with embedded hard magnetic particles," J. Intell. Mater. Syst. Struct., vol. 23, no. 9, pp. 1049-1054, Jun. 2012.

[8] W. Li and X. Zhang, "Research and Applications of MR Elastomers," Recent Patents Mech. Eng., vol. 1, no. 3, pp. 161-166, 2008.

[9] G. J. Liao, X.-L. Gong, S. H. Xuan, C. J. Kang, and L. H. Zong, "Development of a real-time tunable stiffness and damping vibration isolator based on magnetorheological elastomer," J. Intell. Mater. Syst. Struct., vol. 23, no. 1, pp. 25-33, Jan. 2012.

[10] S. Abramchuk et al., "Novel highly elastic magnetic materials for dampers and seals: Part I. Preparation and characterization of the elastic materials," Polym. Adv. Technol., vol. 18, no. 11, pp. 883-890, Nov. 2007.

[11] W. Li, K. Kostidis, Xianzhou Zhang, and Yang Zhou, "Development of a force sensor working with MR elastomers," in 2009 IEEE/ASME International Conference on Advanced Intelligent Mechatronics, 2009, pp. 233-238.

[12] W. . Li, T. F. Tian, and H. Du, Smart Actuation and Sensing Systems - Recent Advances and Future Challenges. Intech, 2012.

[13] M. Lokander and B. Stenberg, "Performance of isotropic magnetorheological rubber materials," Polym. Test., vol. 22, no. 3, pp. 245-251, May 2003.

[14] L. Chen, X. L. Gong, and W. H. Li, "Microstructures and viscoelastic properties of anisotropic magnetorheological elastomers," Smart Mater. Struct., vol. 16, no. 6, pp. 2645-2650, Dec. 2007.

[15] B. Nayak, S. K. Dwivedy, and K. S. R. K. Murthy, "Fabrication and characterization of magnetorheological elastomer with carbon black," J. Intell. Mater. Syst. Struct., vol. 26, no. 7, pp. 1-10, 2014.

[16] L. Chen, X. Gong, W. Jiang, J. Yao, H. Deng, and W. Li, "Investigation on magnetorheological elastomers based on natural rubber," J. Mater. Sci., vol. 42, no. 14 , pp. $5483-$ 5489, Jul. 2007.

[17] M. Farshad and A. Benine, "Magnetoactive elastomer composites," Polym. Test., vol. 23, no. 3, pp. 347-353, May 2004.

[18] W. H. Li and M. Nakano, "Fabrication and characterization of PDMS based magnetorheological elastomers," Smart Mater. Struct., vol. 22, no. 5, p. 055035, May 2013.

[19] B. Ju et al., "Dynamic mechanical properties of magnetorheological elastomers based on polyurethane matrix," Polym. Compos., vol. 37, no. 5, pp. 1587-1595, May 2016.

[20] Y. C. Fan, X. L. Gong, W. Q. Jiang, W. Zhang, B. Wei, and W. H. Li, "Effect of maleic anhydride on the damping property of magnetorheological elastomers," Smart Mater. Struct., vol. 19, no. 5, p. 055015, May 2010.

[21] J. M. Ginde et al., "Magnetorheological Elastomers: Properties and Applications," in SPIE Conference on Smart Materials Technology, 1999, vol. 3675, no. March, pp. 131-138.

[22] I. A. Perales-Martínez, L. M. Palacios-Pineda, L. M. Lozano-Sánchez, O. Martínez-Romero, J. G. PuenteCordova, and A. Elías-Zúñiga, "Enhancement of a magnetorheological PDMS elastomer with carbonyl iron particles," Polym. Test., vol. 57, no. 57, pp. 78-86, Feb. 2017.

[23] Y. Wang et al., "Effects of rubber/magnetic particle interactions on the performance of magnetorheological elastomers," Polym. Test., vol. 25, pp. 262-267, 2006.

[24] J. Wu, X. Gong, Y. Fan, and H. Xia, "Anisotropic polyurethane magnetorheological elastomer prepared through in situ polycondensation under a magnetic field," Smart Mater. Struct., vol. 19, no. 10, p. 105007, Oct. 
2010 .

[25] J. Li, X. Gong, Z. Xu, and W. Jiang, "The effect of prestructure process on magnetorheological elastomer performance," Int. J. Mater. Res., vol. 99, no. 12, pp. 1358-1364, Dec. 2008.

[26] E. Chirila, I. Chirica, D. Boazu, and E. F. Beznea, "MRE Damping Characteristics Evaluation," Key Eng. Mater., vol. 699, pp. 31-36, Jul. 2016.

[27] L. Chen, X. Gong, and W. Li, "Damping of Magnetorheological Elastomers," Chinese J. Chem. Phys., vol. 21, no. 6, pp. 581-585, Dec. 2008.

[28] H. L. Sun, P. Q. Zhang, X. L. Gong, and H. B. Chen, "A novel kind of active resonator absorber and the simulation on its control effort," J. Sound Vib., vol. 300, no. 1-2, pp. 117-125, Feb. 2007.

[29] X. Qiao, X. Lu, X. Gong, T. Yang, K. Sun, and X. Chen, "Effect of carbonyl iron concentration and processing conditions on the structure and properties of the thermoplastic magnetorheological elastomer composites based on poly(styrene-b-ethylene-co-butylene-b-styrene) (SEBS)," Polym. Test., vol. 47, no. 47, pp. 51-58, Oct. 2015.

[30] A. Boczkowska and S. Awietjan, "Microstructure and Properties of Magnetorheological Elastomers," in
Advanced Elastomers - Technology, Properties and Applications, 2012, pp. 147-180.

[31] J. S. An, S. H. Kwon, H. J. Choi, J. H. Jung, and Y. G. Kim, "Modified silane-coated carbonyl iron/natural rubber composite elastomer and its magnetorheological performance," Compos. Struct., vol. 160, no. 160, pp. 1020-1026, Jan. 2017.

[32] J. M. Ginde, M. E. Nichols, L. D. Eliea, and I. L. Tardiff, "Magnetorheological Elastomers: Properties and Applications," in SPIE Conference on Smart Materials Technology, 1999, vol. 3675, no. March, pp. 131-138.

[33] A. V. Chertovich, G. V. Stepanov, E. Y. Kramarenko, and A. R. Khokhlov, "New Composite Elastomers with Giant Magnetic Response," Macromol. Mater. Eng., vol. 295, no. 4, pp. 336-341, Apr. 2010.

[34] N. A. Yunus et al., "Rheological properties of isotropic magnetorheological elastomers featuring an epoxidized natural rubber," Smart Mater. Struct., vol. 25, no. 10, p. 107001, Oct. 2016.

[35] X. L. Gong, X. Z. Zhang, and P. Q. Zhang, "Fabrication and characterization of isotropic magnetorheological elastomers," Polym. Test., vol. 24, pp. 669-676, 2005. 